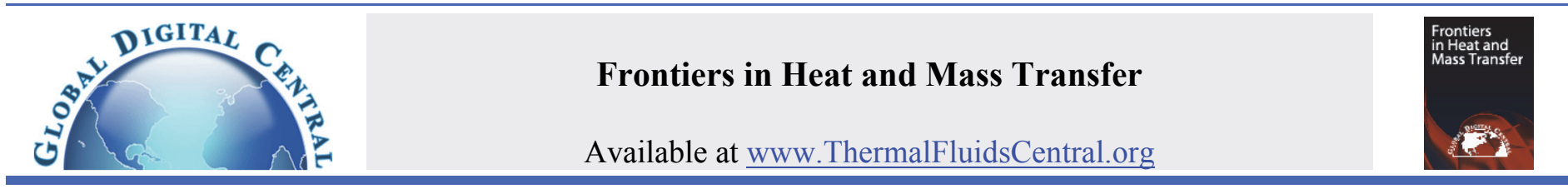

\title{
EFFECT OF SPACESHIP ORBITAL TRANSFER ON SOLUTION CONVECTION DURING PROTEIN CRYSTAL GROWTH UNDER MICROGRAVITY
}

\author{
Kun Zhang* Liang Bi Wang \\ Key Laboratory of Railway Vehicle Thermal Engineering, Lanzhou Jiaotong University, Lanzhou, Gansu, 730070, China
}

\begin{abstract}
Detailed numerical analysis is presented for the effect of spaceship orbital transfer on solution convection during protein crystal growth under microgravity. The results show that the flow and mass transfer during protein crystal growth are unsteady in the process of orbital transfer. For the case of quasi-steady acceleration, the flow is so weak that the effect of flow on concentration field can be negligible. For the case of position adjustment, the convection is enhanced with protein crystal diameter $d_{c}>0.2 \mathrm{~mm}$ and slightly alters the purely diffusive concentration distribution under zero gravity condition. For the case of motor working, the solute transport is mainly controlled by convection and the concentration field near the crystal surface is altered obviously.
\end{abstract}

Keywords: orbital transfer; natural convection; motor working; position adjustment.

\section{INTRODUCTION}

Solution convection is one of the most import factors for the growth of protein crystal. Recently, many researchers studied the effect of solution convection on the crystal growth rate and quality. Wang et al. (2008) studied a dimensionless number to evaluate the convection effects on protein crystal growth. Their results indicated that the growth rate of protein crystal is due to the coupling of convective and diffusive mass transport. Szurgot and Sangwai (1987) studied the influence of free convection on the formation of growth defects in potassium bichromate crystals grown from aqueous solutions. Nanev and Penkova et al. (2004) investigated the effects of buoyancy-driven convection on nucleation and growth of protein crystals. Vekilov et al. $(1998 ; 1999)$ studied the influences of convective solute and impurity transport on protein crystal growth. The convective enhancement of impurity supply can increase the amplitude of defects-causing fluctuations and lead to the crystal quality degradation. Chen and Wang et al. (2013) investigated the effect of buoyancy-driven convection on the needle-like interface shape of the crystal. Many helpful results from the above research of protein growth indicated that convection can affect the crystal growth rate and quality, except for very low flow rates.

Natural convection caused by gravity is a recurrent phenomenon in the world (Li et al., 2014). Studies of solution convection on earth during protein crystal growth have attracted increasing interest over the last few decades due to a desire to improve the growth rate and quality of protein crystals. Lima and Wit (2004) investigated the convective instability in protein crystal growth on the basis of diffusion-convection evolution equations for the concentrations coupled to the Navier-Stokes equations. Lin et al. (1995) studied the time-dependent diffusive-convective transport in an isothermal protein crystal growth system at standard and zero gravity. Qi and Wakayama (2000) investigated numerically the sedimentation flow and buoyancy-driven during protein crystal growth in which the crystal size changes from 0.1 to $100 \mu \mathrm{m}$ and clarified how gravitational fields affect the various stages of crystal growth. Baird and Guo (1998) studied the free convection and surface kinetics in crystal growth from solution. Riahi and Obare (2009) studied the rotating flow around a growing protein crystal. The application of both a magnetic field and a rotation vector on the flow during the protein crystal growth could lead to a more effective procedure to control the strength of the flow and thereby producing higher quality protein crystal. Verma and Srivastava et al. (2005) investigated experimentally and numerically the solute convection in the initial stages of crystal growth from an aqueous solution.

Gravity is generally considered unfavorable since it gives rise to convection during the protein growth. Microgravity is believed to an ideal environment to obtain high quality crystals due to the diffusive transport domination that allows a good arrangement of the monomers in the crystal lattice and increasing the final quality (Takahashi et al., 2013). However, past experience has also shown that the microgravity environment on the Space Shuttle can sometimes yield unpredictable results in PCG. The data collected from past shuttle missions have all indicated that about only $35 \%$ of crystal grown in space better than the best earth grown samples (Kundrot et al., 2001). In fact, microgravity is not zero gravity. The residual gravity or the so-called g-jitter on spaceship causes unsteady natural convection flow, thus affecting the growth rate and quality of protein crystals (Simic-Stefani et al., 2006; Bauer, 1984). Many studies have been conducted on the solution convection during protein crystal growth under microgravity. Ramachandran et al. (1995) studied the analytical and numerical models of flows and transport in protein crystal growth under the influence of steady, transient, and periodic accelerations at various levels. Feonychev and Dolgikh (2001) investigated the effects of constant and variable accelerations on the melt flow and heat and mass transfer in the process of crystals growth on board spacecraft by the method of directional crystallization. Carotenuto and Cartwright et al. (2002) studied the solutal buoyancy-driven convection and sedimentation caused by the different densities of the crystal and the protein solution in microgravity. 
Tanaka and Sasaki et al. (2013) investigated the numerical model of protein crystal growth in a diffusive field such as the microgravity environment. Lan and Tu (2002) investigated the flow and segregation under the control of slow rotation for Bridgman crystal growth in microgravity.

Comparatively, few works have been reported on unsteady convection during protein crystal growth in the process of spaceship orbital transfer, especially for the conditions of real space flight. Yuferev and Kolesnikova (1995a; 1995b) studied numerically the unsteady convection under coactions of residual acceleration and Coriolis force caused by spaceship rotation. Kolesnikova and Polovko et al. (1997) investigated the influence of Coriolis force caused by rotation of an orbital station on thermal convection and impurity segregation during crystal growth under microgravity. Bessonov and Polezhaev (2001) studied numerically the convection in a cylinder sensor using real data on micro accelerations on board the Mir station.

The objective of this paper is to study the effect of spaceship orbital transfer on the natural convection during protein crystal growth under microgravity. The governing equations will be discretized using the finite volume method based on staggered grid formulation, and solved using the SIMPLE algorithm with QUICK scheme. The flow and concentration fields caused by different micro-gravitational accelerations level will be numerically simulated. The Peclet number $(\mathrm{Pe})$ based on the relative magnitude of convection will be studied to investigate the effect of microgravity acceleration caused by orbital transfer on the solution convection and concentration fields.

\section{PROBLEM FORMULTION}

A schematic diagram of the physical model under consideration is shown in Figure 1. We consider a cylindrical crystal was growing from protein solutions in a cylindrical container. The crystal was sediment to and located at the bottom of the container.

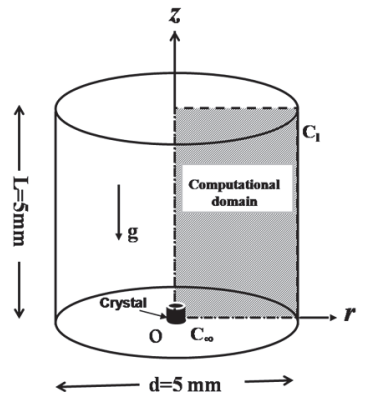

Fig. 1 Physical model

The inner crystal surface and outer container walls are kept uniform except for different concentration $C_{l}$ and $C_{\infty}$, respectively, with $C_{l}<C_{\infty}$. The concentration differences between the two cylinders lead to natural convection. It is assumed that the fluid in the enclosure is of a Boussinesq type, and the flow and mass transfer is two-dimensional and laminar. The governing equations for flow and solute concentration are Equation of continuity:

$\frac{1}{r} \frac{\partial r V_{r}}{\partial r}+\frac{\partial V_{z}}{\partial z}=0$

Equation of motion:

$\rho \frac{\partial V_{r}}{\partial t}+\rho V_{r} \frac{\partial V_{r}}{\partial r}+\rho V_{z} \frac{\partial V_{r}}{\partial z}=-\frac{\partial p}{\partial r}+\rho v\left[\frac{1}{r} \frac{\partial}{\partial r}\left(r \frac{\partial V_{r}}{\partial r}\right)+\frac{\partial^{2} V_{r}}{\partial z^{2}}-\frac{V_{r}}{r^{2}}\right]$

$\rho \frac{\partial V_{z}}{\partial t}+\rho V_{r} \frac{\partial V_{z}}{\partial r}+\rho V_{z} \frac{\partial V_{z}}{\partial z}=-\frac{\partial p}{\partial z}+\rho v\left[\frac{1}{r} \frac{\partial}{\partial r}\left(r \frac{\partial V_{z}}{\partial r}\right)+\frac{\partial^{2} V_{z}}{\partial z^{2}}\right]-\rho g$

Equation of dimensionless concentration:

$\frac{\partial \varphi}{\partial t}+(V \cdot \nabla) \varphi=D_{s}\left(\nabla^{2} \varphi\right)$
Where $V, \phi, p, t, \rho$ and $\mathrm{g}$ are the velocity vector, dimensionless concentration, pressure, time, density of the solution and microgravitational acceleration, respectively. $D_{s}=7 \times 10^{-11}$ is the diffusion coefficient $\left(\mathrm{m}^{2} / \mathrm{s}\right), v=10^{-6}$ is the kinematic viscosity $\left(\mathrm{m}^{2} / \mathrm{s}\right)$.

The density difference of the solution is approximately proportional to the difference in the protein concentration. Therefore, the density of the solution can be written as:

$\rho=\rho_{l}\left(1+\Gamma_{S} \varphi\right)$

The dimensionless parameters used in Eqs. (4) (5) are defined as follows.

$\Gamma_{S}=\frac{\rho_{\infty}-\rho_{l}}{\rho_{l}}, \varphi=\frac{C-C_{l}}{C_{\infty}-C_{l}}$

where $\Gamma \mathrm{s} \approx 0.00795$ is the nominal diffusion coefficient, $\rho_{l}=1007 \mathrm{~kg} / \mathrm{m} 3$ is the density of the crystal interface $\left(\mathrm{kg} / \mathrm{m}^{3}\right), C$ is the concentration of solution. The subscripts of 1 and $\infty$ represent the crystal interface and a position far from the crystal, respectively.

When the spaceships move from one orbit to another, the quasisteady acceleration on the "Shenzhou" series of spaceships can be analyzed, and the transient accelerations can be detected by the quartz flexible accelerometer (Xue, 2004). For the case of orbital transfer, the micro-gravitational accelerations (g) include quasi-steady acceleration and two types of transient accelerations, as is shown in Fig. 2.

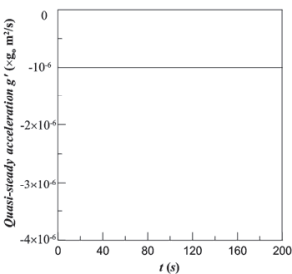

(a) $g^{\prime}$

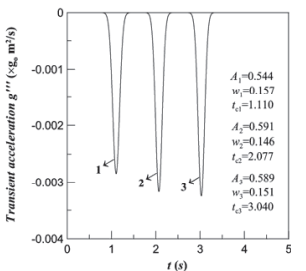

(b) g"

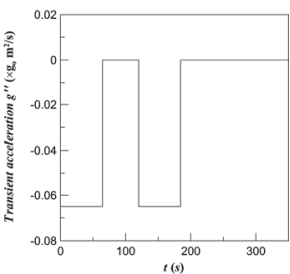

(c) $g^{\prime \prime \prime}$
Fig. 2 Three types of microgravity accelerations for orbital transfer

The quasi-steady acceleration $\left(\mathrm{g}^{\prime}\right)$ in Fig. 2(a) is due to atmospheric drag, tide forces, solar radiation pressure. The first type of transient accelerations ( $\left.\mathrm{g}^{\prime \prime}\right)$ in Fig. 2(b) and the second type of transient acceleration ( $\left.\mathrm{g}^{\prime \prime \prime}\right)$ in Fig. 2(c) are related to motor working and position adjustment respectively. Therefore, the micro-gravitational acceleration can be written as

$g=g^{\prime}+g^{\prime \prime}+g^{\prime \prime \prime}$

The initial conditions are

$t=0, V_{r}=0, V_{z}=0, \phi=1$

The growth velocity is sufficiently low that a moving boundary can be safely ignored. No-slip flow conditions are imposed on the surface of the crystal and on the container walls. Therefore, the boundary conditions for Eqs. (1)- (4) are

$$
\begin{aligned}
& r=0, \frac{\partial V_{r}}{\partial r}=0, \frac{\partial V_{z}}{\partial r}=0, \frac{\partial \phi}{\partial r}=0 \\
& r=d / 2, V_{r}=0, V_{z}=0, \phi=1 \\
& z=0, \quad V_{r}=0, V_{z}=0, \frac{\partial \phi}{\partial z}=0 \\
& z=L, \quad V_{r}=0, V_{z}=0, \phi=1
\end{aligned}
$$


To evaluate the effect of buoyancy driven convection on solute transport, the solute Peclet number based on the relative magnitude of convective to diffusive transports can be defined by Ref. [13].

$P e=V_{\max } \frac{d_{c}}{2 D_{s}}$

where $d_{c}$ is the diameter of protein crystal and $V_{\max }$ is the maximum velocity of solution convection.

\section{NUMERICAL PROCEDURES}

For the study of solution convection during protein crystal growth under microgravity, the numerical code is based upon a finite volume method with explicit time steps and a semi-iterative pressure-velocity correction (SIMPLE algorithm). The governing equations were discretized on a staggered mesh, and the central difference scheme was adopted for the discretization of the diffusion terms. To achieve high resolution of the velocity and concentration fields near the crystal interface, the grid lines are more closely packed near the crystal surface than in the bulk. Figure 3 shows the two samples of grid system near the protein crystal for the different locations in this study.

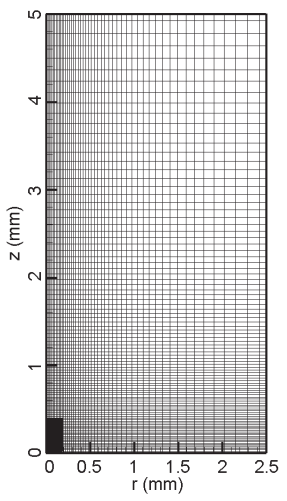

(a) crystal at the bottom

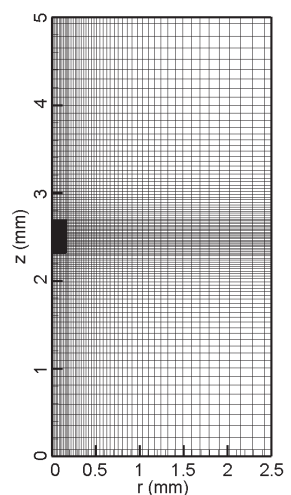

(b) crystal suspended
Fig. 3 Sample of computational grid near the crystal

For the case of crystal size $d_{c}=0.1 \mathrm{~mm}$, the grid-independence of the numerical results is studied for the case of protein crystal growth under constant gravity. When the time step is equal to 0.001 , the five mesh sizes in Table 1 are used to complete the grid-independence study. It is noted that the total numbers of grid points for the above five size are $50 \times 100$, $70 \times 140,90 \times 180,100 \times 200$, and $150 \times 300$, respectively. Numerical experiments showed that the relative error of maximum velocity at $t=20 \mathrm{~s}$ corresponding to a grid number of and is less than $1 \% ; 100 \times 200$ grid points are adequate to yield accurate results. Table 2 shows that the maximum velocity at $t=20 \mathrm{~s}$ of different time steps for $100 \times 200$ grid points. The difference between Vmax obtained using the time steps of 0.001 and 0.0001 is negligible. So the time step is selected as 0.001 for the present computations. When the crystal size are larger than $0.1 \mathrm{~mm}$, the number of grid points in the calculation are the same as the number of the corresponding grid points for $d_{c}=0.1 \mathrm{~mm}$. The grid independence of the numerical results for $d_{c}>0.1 \mathrm{~mm}$ can be still guaranteed due to the decrease of flow space. Therefore, the selected numbers of grid points are $100 \times 200$ grid points for all the crystal sizes. For validation of the results obtained in this study, the flow and dimensionless concentration fields for the case of crystal size $d_{c}=0.1 \mathrm{~mm}$ are investigated and compared with those obtained by Qi et al. (2000). The values of maximum velocities at the constant gravity $\left(g_{0}=9.8 \mathrm{~m} 2 / \mathrm{s}\right)$ are equal to $62.08 \mu \mathrm{m} / \mathrm{s}$ and $62.7 \mu \mathrm{m} / \mathrm{s}$ respectively. The relative difference of maximum velocities is less than $1 \%$. The dimensionless concentrations in the solution along the radial axis at the central of protein crystal are also calculated numerically in Figure 4 . The computational results indicate that the numerical results agreed very well with the results in reference.
Table 1 Maximum velocities at $t=20$ s for different grid numbers

\begin{tabular}{|l|l|l|l|l|l|}
\hline Grid & $50 \times 100$ & $70 \times 140$ & $90 \times 180$ & $100 \times 200$ & $150 \times 300$ \\
\hline$V_{\max }$ & $6.426 \mathrm{e}-5$ & $6.362 \mathrm{e}-5$ & $6.316 \mathrm{e}-5$ & $6.260 \mathrm{e}-5$ & $6.204 \mathrm{e}-5$ \\
\hline
\end{tabular}

Table 2 Maximum velocities at $t=20$ s for different time steps

\begin{tabular}{|c|c|c|c|c|c|}
\hline Time step & 0.05 & 0.01 & 0.005 & 0.001 & 0.0001 \\
\hline$V_{\max }$ & $5.029 \mathrm{e}-5$ & $6.131 \mathrm{e}-5$ & $6.224 \mathrm{e}-5$ & $6.260 \mathrm{e}-5$ & $6.249 \mathrm{e}-5$ \\
\hline
\end{tabular}

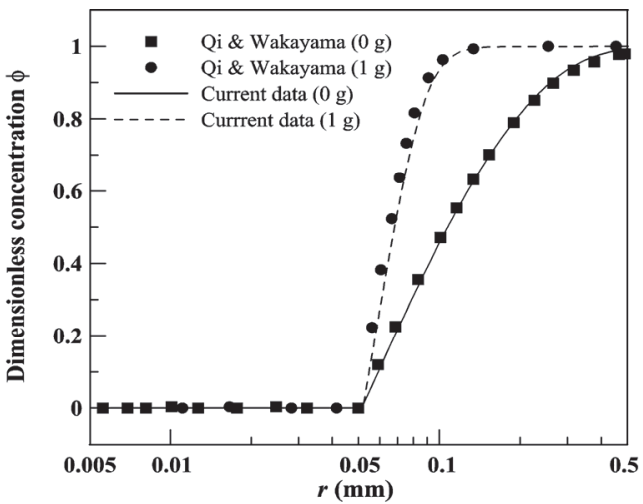

Fig. 4 Comparison of current and previous numerical results for $d_{c}=0.1$ $\mathrm{mm}$

\section{CITATIONS AND LIST OF REFERENCES}

\subsection{Effect of quasi-steady acceleration}

Quasi-steady accelerations are those that vary little over long periods of time and estimated to be on the order of $10^{-6} \mathrm{~g}_{\mathrm{o}}$. Numerical simulations of the natural convection in the crystal size of $0.1 \mathrm{~mm}-0.8 \mathrm{~mm}$ are performed for the crystal suspended in the center and the crystal at the bottom of the container. Figure 5 shows the dimensionless concentration fields under zero gravity for different locations and crystal sizes. The effects of the quasi-steady acceleration on the dimensionless concentration were obtained as shown in Fig. 6. For the case of crystal size $d_{c}=0.8 \mathrm{~mm}$, the values of maximum velocity are $7.84 \times 10^{-7} \mathrm{~mm} / \mathrm{s}$ and $1.09 \times 10^{-6} \mathrm{~mm} / \mathrm{s}$ for the crystal at the bottom or suspended in the container, respectively. For the case of quasi-steady acceleration, the solution convections are sufficiently small, diffusive transport still dominates, the dimensionless concentration distribution is not changed by convection and is similar to that under zero gravity.

When the crystals are at the bottom of containers, the values of Peclet numbers are equal to $1.17 \times 10^{-4}$ for $d_{c}=0.1 \mathrm{~mm}$ and $8.96 \times 10^{-4}$ for $d_{c}=0.8 \mathrm{~mm}$, respectively. When the crystals suspended in the center of containers, the values of Pe are equal to $3.31 \times 10^{-4}$ for $d_{c}=0.1 \mathrm{~mm}$ and $1.24 \times 10^{-2}$ for $d_{c}=0.8 \mathrm{~mm}$, respectively. In this scope of the crystal size from $0.1 \mathrm{~mm}$ to $0.8 \mathrm{~mm}$, the value of Pe for the case of crystal suspended is greater than that for the case of crystal at the bottom. The effect of quasi-steady acceleration on the growth of protein crystal suspended in the center of the container is weaker than that at the bottom of container. More numerical simulation indicated that an increase in crystal sizes lead to the increase of the value of Pe. However, all the values of Peclet numbers under quasi-steady acceleration condition are less than 1 . The results indicate that the effect of quasi-steady acceleration under microgravity on the growth of protein crystal growth is small enough, and it can be ignored.

\subsection{The effect of transient acceleration caused by position adjustment}

When the spaceship transfers from one orbit to another, the transfer can be also accomplished with position adjustment many times. For one position adjustment, the data of micro-gravitational level on the "Shenzhou" spaceship can be detected by a quartz flexible accelerometer. The measure data from the reference can be fitted for the gauss 
polynomial with three peaks by Gaussian curve-fitting method (Xue et al., 2004). The gauss polynomial for micro-gravitational acceleration can be written as follows.

$$
g=g^{*}+\frac{A}{w \sqrt{\pi / 2}} g_{o} \exp \left[-\frac{2\left(t-t_{c}\right)^{2}}{w^{2}}\right]
$$

where $g^{*}, A, w$ and $t_{c}$ are the zero baseline, peak area, width and location, respectively. The Gaussian fitting curve and the values of parameters for three fitting peaks are shown in Figure 2(b).
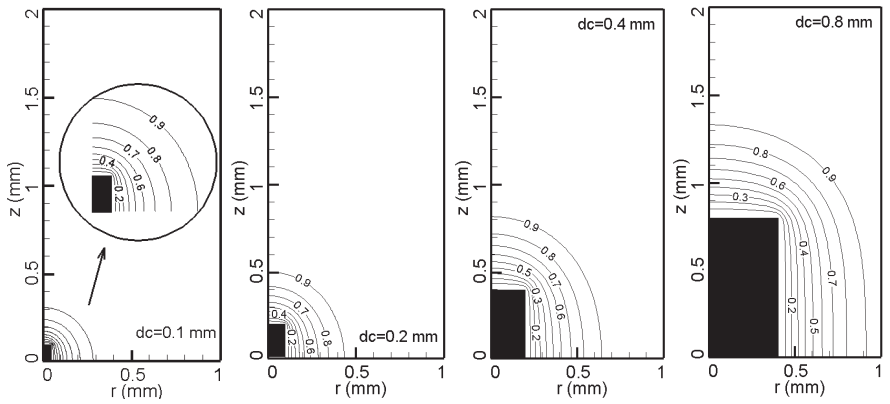

(a) crystal at the bottom of the container
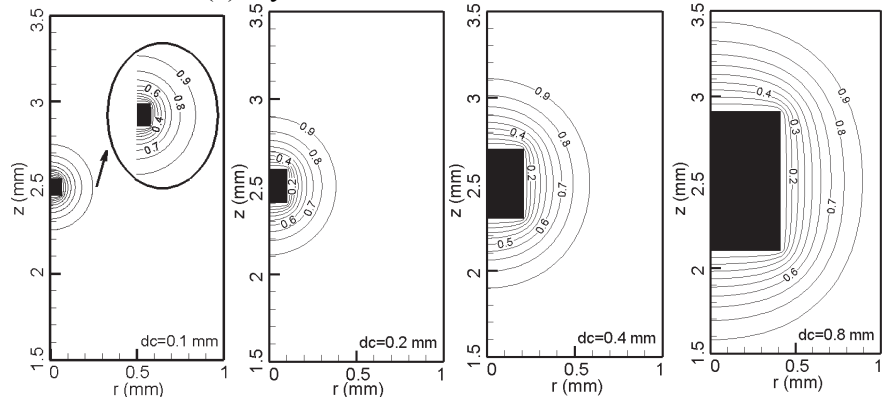

(b) crystal suspended in the container

Fig. 5 Dimensionless concentration fields under zero gravity conditions for different crystal sizes

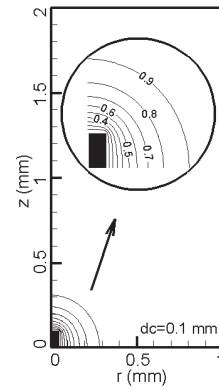

(a) crystal at the bottom

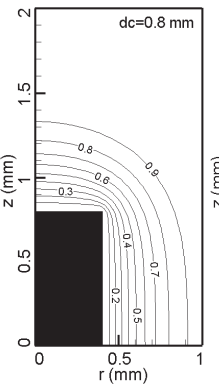

m

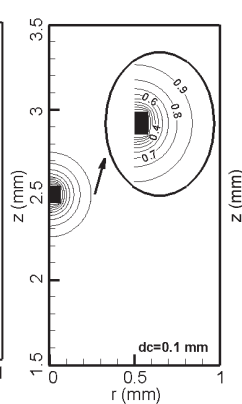

(b) crystal suspended

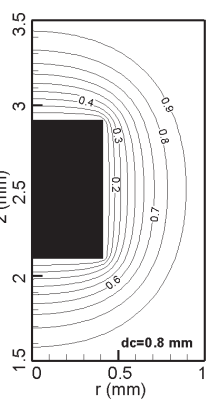

nder quasi-steady acceleration conditions for crystal suspended in the container.

A simulation of natural convection caused by position adjustment is performed from the initial steady velocity and concentration under zerogravity. For the case of $d_{c}=0.4 \mathrm{~mm}$, the value of maximum velocity with different time is shown in Fig. 7(a). Two points on top right side of the crystal for $(73,144)$ and $(59,164)$ were selected to describe the change of dimensionless concentration for the crystal at the bottom and suspended, respectively (see Figure 7(b)). When the level of transient acceleration under microgravity environment increased, the value of maximum velocity also increased rapidly over $0.35 \times 10^{-3} \mathrm{~mm} / \mathrm{s}$ and the value of Pelect number is greater than 1 . The convection caused by position adjustment becomes obvious in quite a short time and begins to influence the concentration distribution. Almost at the same time, the value of dimensionless concentration at monitor point decreased rapidly due to the convection. Then the value of maximum velocity decreased rapidly with the decreasing level of micro-gravitational acceleration, and soon it is lower than $0.35 \times 10^{-3} \mathrm{~mm} / \mathrm{s}$. A decrease in the value of velocity leads to the decrease of Pelect number. When the Pelect number is lower than 1, the flow becomes so weak that it cannot influence the concentration distribution obviously, and the solute transport controlled by diffusion is quite slowly. Therefore, the value of dimensionless concentration seems to be constant for a time. In the duration of time from $t=0 \mathrm{~s}$ to $t=5 \mathrm{~s}$, the above process can be repeated three times for a position adjustment. An increase or decrease in the level of microacceleration can lead to the increase or decrease of the value of $V_{\max }$. When the crystal are at the bottom of the container and suspended in the container, the maximum peak values of $V_{\max }$ are $0.897 \times 10^{-3} \mathrm{~mm} / \mathrm{s}$ and $1.148 \times 10^{-3} \mathrm{~mm} / \mathrm{s}$ at about $t=3.08 \mathrm{~s}$, respectively. For the case of suspended crystal, the sustained time for Pe $>1$ is about 0.486 second, which is longer than 0.347 second for the crystal at the bottom. The flow for the crystal at the bottom is weaker than that for the suspended crystal. Figure 8 shows the time signal of Pelect numbers for different sizes during position adjustment. It can be seen that with the increasing crystal size, the sustained time for $\mathrm{Pe}>1$ becomes longer and the value of $\mathrm{Pe}$ also increases. The numerical investigation revealed that for the case of $d_{c}<0.2$, the convection caused by position adjustment is so weak that the change of concentration field caused by this convection is negligible. When the crystal size is greater than $0.2 \mathrm{~mm}$, the flow caused by position adjustment is enhanced and slightly alters the purely diffusive concentration distribution under zero gravity condition.

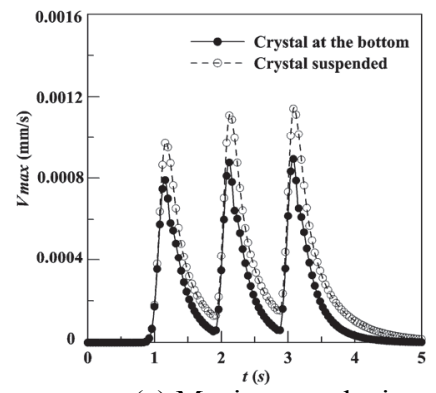

(a) Maximum velocity

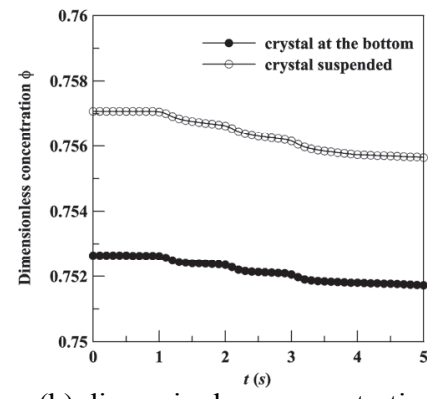

(b) dimensionless concentration
Fig. 7 Time signal of maximum velocity and dimensionless concentration at the point $(0.3 \mathrm{~mm}, 0.6 \mathrm{~mm})$ for $d_{c}=0.4 \mathrm{~mm}$

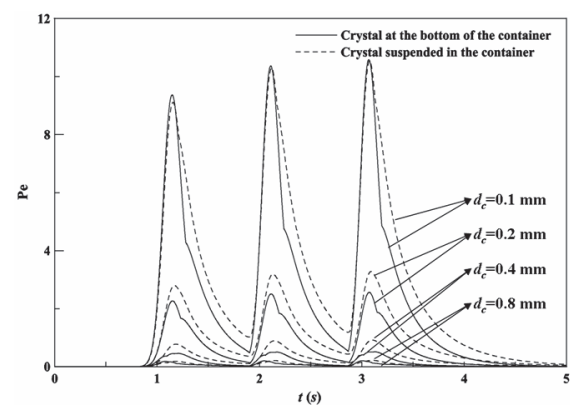

Fig. 8 Time signal of $\mathrm{Pe}$ for different crystal sizes during position adjustment

4.3 The effect of transient acceleration caused by motor working When the spaceship transfers from the initial to final orbit directly, the transfer can be accomplished with a single impulsive disturbance. The measure data on the "Shenzhou" spaceship indicated that the single impulsive disturbance is mainly induced by motor working. The microgravitational level of the single impulse is on the order of $6.5 \times 10^{-2} \mathrm{~g}_{\mathrm{o}}$ and the sustained time is $64.37 \mathrm{~s}$, which is recorded by Xue et al. (2004). Numerical simulations of the natural convection caused by motor working are performed from the initial velocity and concentration fields under zero-gravity. When the protein crystal with $d_{c}=0.4 \mathrm{~mm}$ are at the bottom of the container, the flow and concentration fields at different time are obtained as shown in Fig. 9 and Fig. 10, respectively. At time $t$ $=1 \mathrm{~s}$, a single vortex on the lower left occurs, while the isotherms remain the basic shapes for a short time. The vortex grows larger and moves 
toward the left of the container in Fig. 9 (b-c). The isotherms become thick near the right side of crystal, showing the increasing concentration gradient. The value of $V_{\max }$ increases firstly and then decreases gradually with time. The value of Pe remains over 10 in the whole process of motor working nearly. The flow is enhanced and the effects of the flow on concentration fields become more and more obvious. The peak value of maximum velocity is $0.11 \mathrm{~mm} / \mathrm{s}$ at time $\mathrm{t}=24.28$. The vortex center occurs nearly at the center of r-axis, and the isotherms alter obviously as are depicted in Fig. 9 (c) and Fig. 10 (c). The vortex moves up progressively, while the value of maximum velocity decreases gradually with time. In comparison with growth under zero gravity condition, the concentration field near the crystal surface is greatly distorted by convection. Afterwards, the transient acceleration caused by motor working disappears at $\mathrm{t}=64.37 \mathrm{~s}$. In the duration of time from $64.37 \mathrm{~s}$ to $65.37 \mathrm{~s}$, the value of maximum velocity decreases dramatically, and the flow becomes sufficiently small such that $\mathrm{Pe}<1$. The concentration field alters slowly due to the solute transport controlled by diffusion after 65.37 s, as is shown in Fig. 10. Finally, the isotherms recover original shape over a long time span.

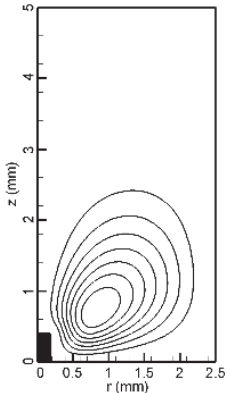

(a) $t=1 \mathrm{~s}$

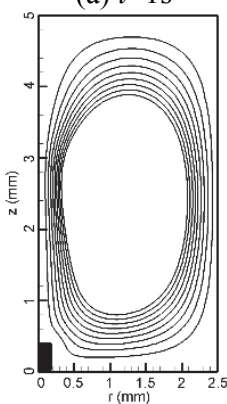

(d) $t=40 \mathrm{~s}$

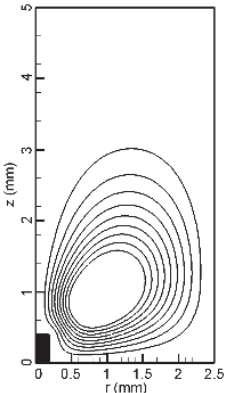

(b) $t=10 \mathrm{~s}$

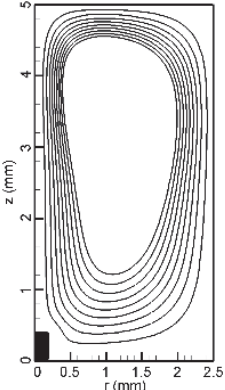

(e) $t=64.37 \mathrm{~s}$

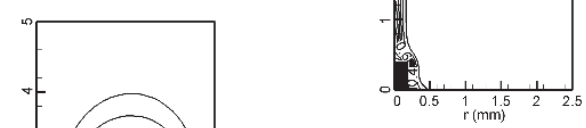

(e) $t=64.37 \mathrm{~s}$

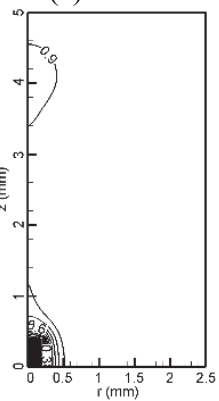

(i) $t=300 \mathrm{~s}$

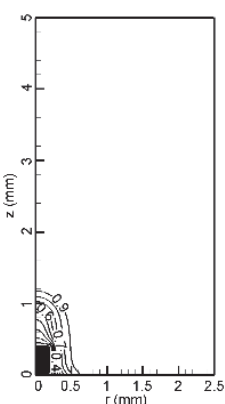

(b) $t=10 \mathrm{~s}$

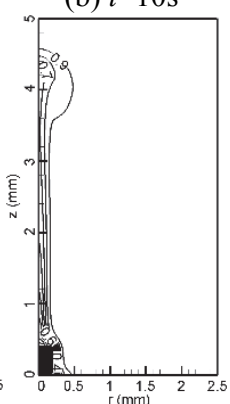

(f) $t=66.37 \mathrm{~s}$

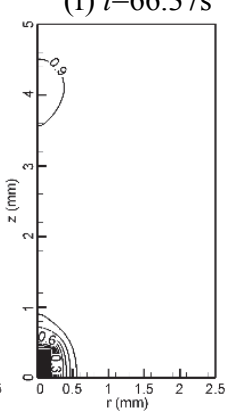

(g) $t=400 \mathrm{~s}$

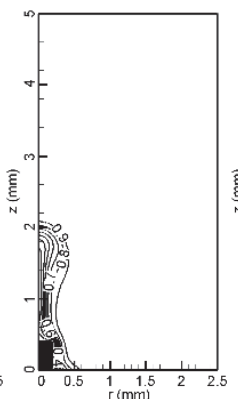

(c) $t=24.28 \mathrm{~s}$

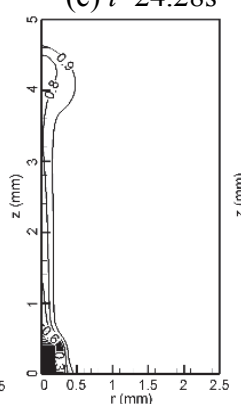

(g) $t=100 \mathrm{~s}$

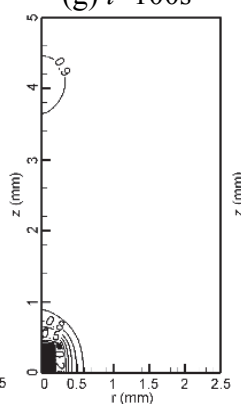

(k) $t=500 \mathrm{~s}$

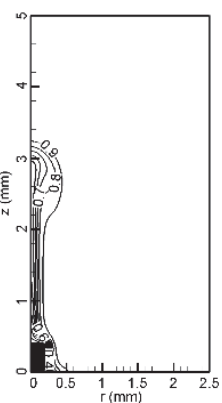

(d) $t=40 \mathrm{~s}$

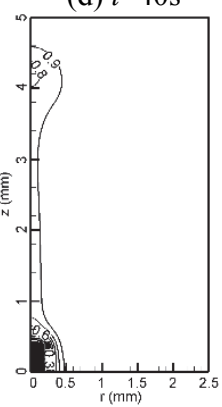

(h) $t=200 \mathrm{~s}$

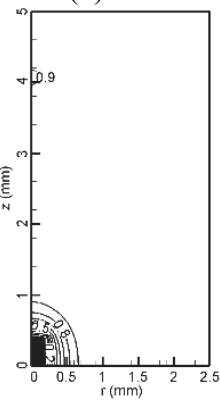

(1) $t=800 \mathrm{~s}$
Fig. 10 Dimensionless concentration fields at different time for the crystal at the bottom of the container

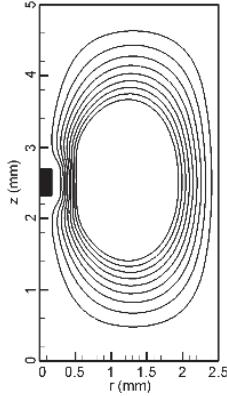

(a) $t=1 \mathrm{~s}$

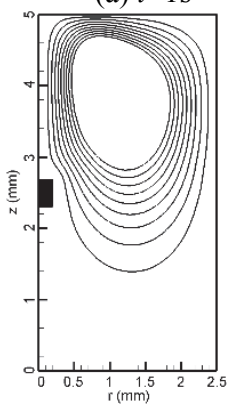

(d) $t=40 \mathrm{~s}$

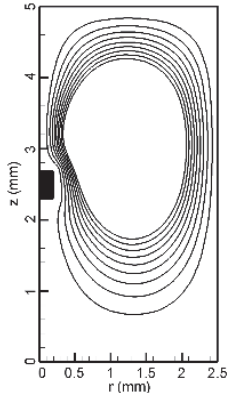

(b) $t=10 \mathrm{~s}$

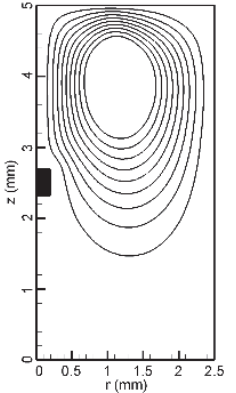

(e) $t=64.37 \mathrm{~s}$

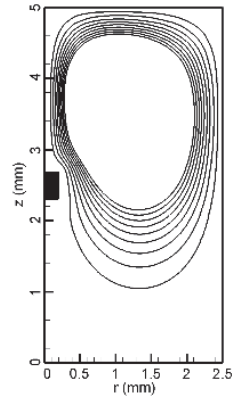

(c) $t=18.74 \mathrm{~s}$

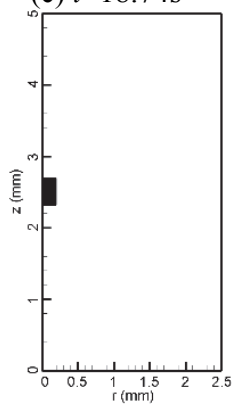

(f) $t=66.37 \mathrm{~s}$ becomes small enough, the isotherms begin to shrink and the concentration field ultimately evolves to initial field for a long time.

Figure 13 shows the time signal of maximum velocity for the crystal size with $d_{c}=0.4 \mathrm{~mm}$. The results indicated that in comparison with the flow for the settled crystal, the flow for the suspended crystal is stronger at the beginning, and it becomes weaker in the later part. The authors
Fig. 11 Flow fields at different time for the crystal suspended in the container.

believe that the reason for this phenomenon is related to the formation and development of vortex in the convection. For the suspended crystal, the vortex on the right side of crystal occurs and develops more readily 
at the beginning of the convection, because the development of the vortex is not limited by the lower wall. When the vortex moves up, the large space on the top of the container is important for the development of the vortex. Therefore, the convection for the crystal at the bottom seems to be stronger than that for the crystal suspended in the container.

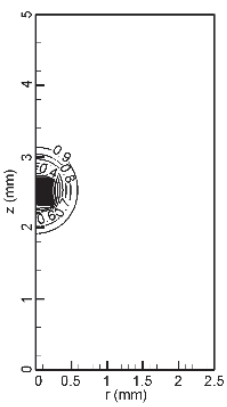

(a) $t=1 \mathrm{~s}$

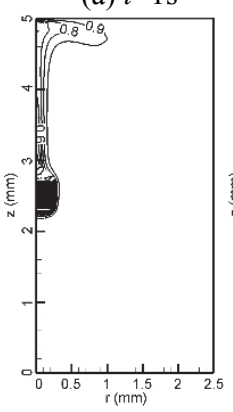

(e) $t=64.37 \mathrm{~s}$

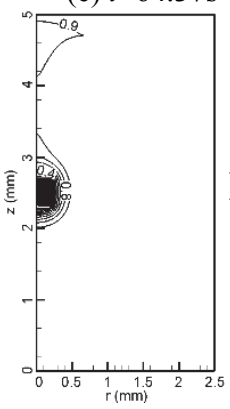

(i) $t=300 \mathrm{~s}$

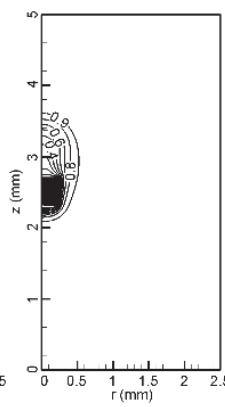

(b) $t=10 \mathrm{~s}$

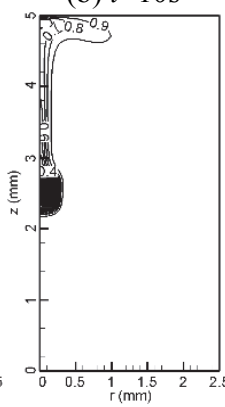

(f) $t=66.37 \mathrm{~s}$

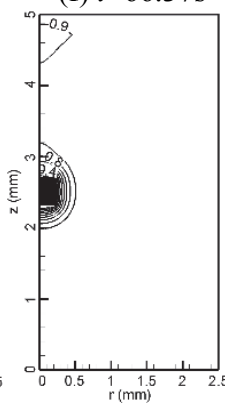

(g) $t=400 \mathrm{~s}$

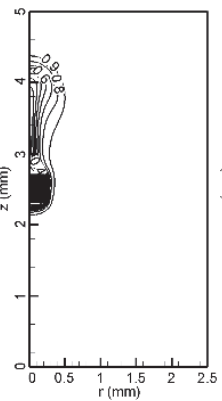

(c) $t=18.74 \mathrm{~s}$

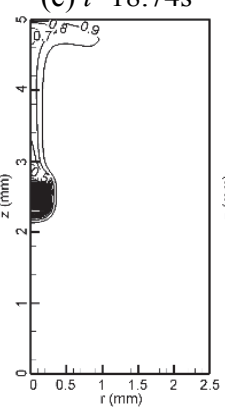

(g) $t=100 \mathrm{~s}$

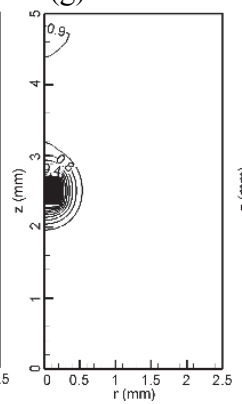

(k) $t=500 \mathrm{~s}$

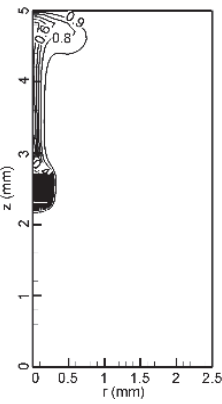

(d) $t=40 \mathrm{~s}$

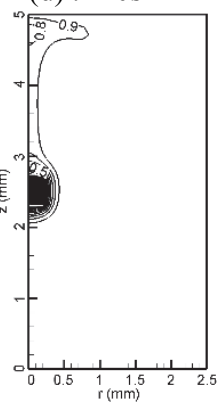

(h) $t=200 \mathrm{~s}$

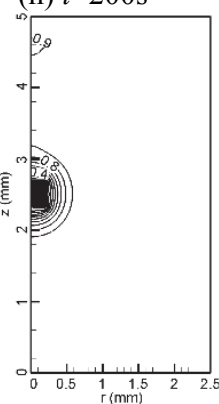

(l) $t=800 \mathrm{~s}$
Fig. 12 Dimensionless concentration fields at different time for the crystal suspended in the container.

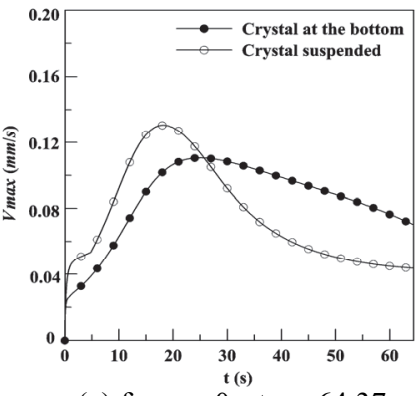

(a) from $t=0 \mathrm{~s}$ to $t=64.37 \mathrm{~s}$

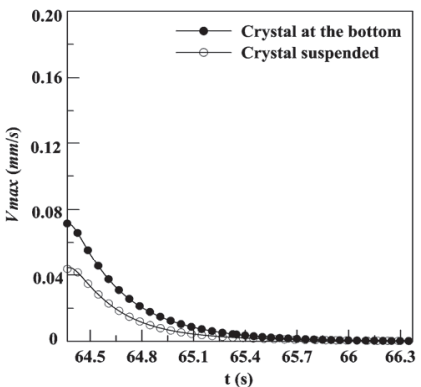

(b) from $t=64.37 \mathrm{~s}$ to $t=66.37 \mathrm{~s}$
Fig. 13 Time signal of maximum velocity for $d_{c}=0.4 \mathrm{~mm}$.

The value of Pelect numbers for different crystal sizes are shown in Fig. 14. For the case of $d_{c}=0.1 \mathrm{~mm}$, the value of Pelect numbers increase with time gradually. When the crystal size is greater than $0.2 \mathrm{~mm}$, the value of Pe increases firstly and then decreases gradually with time. When the transient acceleration caused by motor working exists, the value of Pe remains over ten in the nearly whole process. For the case of motor working, the solute transport is mainly controlled by convection and the concentration field near the crystal is altered significantly. In the scope of crystal size from $0.1 \mathrm{~mm}$ to $0.8 \mathrm{~mm}$, an increase in crystal sizes lead to the increase of Pe. The results show that the flow caused by motor working under microgravity environment is enhanced for the larger crystal size, and the influence of motor working on crystal growth for larger crystal size is more obviously than that for smaller crystal size.

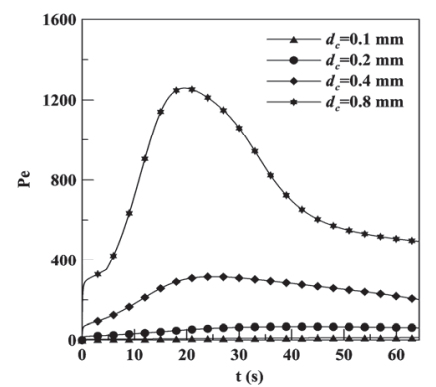

(a) crystal at the bottom

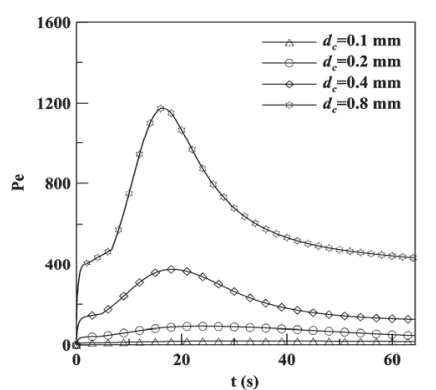

(b) crystal suspended
Fig. 14 Time signal of Pelect numbers for different crystal sizes.

\section{CONCLUSIONS}

The numerical simulations were carried out for the natural convection during protein crystal growth under microgravity environment. The influences of orbital transfer under microgravity environment on the convections are considered. The results indicated that the microgravity level of quasi-steady acceleration, transient accelerations caused by motor working and position adjustment are different in the process of orbital transfer. In the scope of crystal size from $0.1 \mathrm{~mm}$ to $0.8 \mathrm{~mm}$, the flow caused by quasi-steady acceleration is weak and the effect of the flow on concentration field is negligible. For the case of position adjustment, the convection is enhanced with $d_{c}>0.2 \mathrm{~mm}$ and slightly alters the purely diffusive concentration distribution under zero gravity condition. For the case of motor working, the solute transport is mainly controlled by convection and the concentration field near the crystal surface is altered significantly. The solution convections during protein crystal growth caused by motor working are stronger than those caused by position adjustment. The convections for the crystal at the bottom are stronger than that for the crystal suspended in the container. The influence of micro-gravitational acceleration on the solution convection becomes more obvious with the increasing crystal sizes.

\section{ACKNOWLEDGEMENTS}

The financial supports from the Chinese National Natural Science Foundation under Grants No. 51206072 are gratefully acknowledged.

\section{NOMENCLATURE}

A peak area of gauss polynomial (s)

$C \quad$ concentration of solution $(\mathrm{mol} / \mathrm{L})$

$C_{l} \quad$ concentration of inner crystal surface $(\mathrm{mol} / \mathrm{L})$

$C_{\infty} \quad$ concentration of container wall, $\mathrm{mol} / \mathrm{L}$

d diameter of protein crystal

$D_{s} \quad$ diffusion coefficient, $\mathrm{m}^{2} / \mathrm{s}$

$g$ gravitational acceleration, $\mathrm{m} / \mathrm{s}^{2}$

$g^{\prime} \quad$ quasi-steady acceleration

$g^{\prime \prime} \quad$ transient acceleration caused by position adjustment

$g^{\prime \prime \prime} \quad$ transient acceleration caused by motor working

$g_{0} \quad$ gravity under earth's surface, $9.8 \mathrm{~m}^{2} / \mathrm{s}$

$g^{*} \quad$ zero baseline of gauss polynomial

$p$

$P e$

pressure, $\mathrm{Pa}$

$r \quad$ radial coordinate

$t \quad$ time, $\mathrm{s}$

$t_{c} \quad$ location of time, $\mathrm{s}$

$V_{\max } \quad$ maximum velocity of solution convection

$V_{r} \quad$ radial velocity

$V_{z} \quad$ vertical velocity 
w width of gauss polynomial, $\mathrm{s}$

$z \quad$ vertical coordinate

Greek Symbols

$v \quad$ kinematic viscosity $\left(\mathrm{m}^{2} / \mathrm{s}\right)$

$\Gamma \mathrm{s} \quad$ nominal diffusion coefficient

$\rho \quad$ density, $\mathrm{kg} / \mathrm{m}^{3}$

$\rho l \quad$ density of crystal interface, $\mathrm{kg} / \mathrm{m}^{3}$

\section{REFERENCES}

Baird J. K., Guo L., 1998, "Free Convection and Surface Kinetics in Crystal Growth from Solution," Journal of Chemical Physics, 109(6), 2503-2508.

http://dx.doi.org/10.1063/1.476821

Bauer H. F., 1984, "Combined Thermo-capillary- and Natural Convection and g-jitter in a Constant Micro-gravity Field," Forschung im Ingenieurwesen, 50(6), 169-183.

http://dx.doi.org/10.1007/BF02560837

Bessonov O. A., Polezhaev V. I., 2001, "Mathematical Modeling of Convection in the DACON Sensor under Condition of Real Space Flight" Cosmic Research, 39(2), 170-178.

http://dx.doi.org/10.1023/A:1017599228768

Carotenuto L., Cartwright, J. H. E., Castagnolo, D., García Ruiz, J. M., Otálora, F., 2002, "Theory and Simulation of Buoyancy Driven Convection around Growing Protein Crystals in Microgravity," Microgravity Science and Technology, 13(3), 14-21.

http://dx.doi.org/10.1007/BF02872072

Chen M. W., Wang B., Wang Z. D., 2013, "Effect of Buoyancy-Driven Convection on Steady State Dendritic Growth in a Binary Alloy," Chin. Phys. B, 22(11), 116805

http://dx.doi.org/10.1088/1674-1056/22/11/116805

Feonychev A. I., Dolgikh G. A., 2001, "Effects of Constant and Variable Accelerations on Crystals Growth on Board Spacecraft by the Method of Directional Crystallization," Cosmic Research, 39(4), 365-374.

http://dx.doi.org/10.1023/A:1017988514913

Kolesnikova E. N. et al., 1997, "Influence of Coriolos Force on Thermal Convection and Impurity Segregation during Crystal Growth under Microgravity," Journal of Crystal Growth, 180, 578-586.

http://dx.doi.org/10.1016/S0022-0248(97)00268-6

Kundrot C. E. et al., 2001, "Micro-gravity and Macromolecular Crystallography," Crystal Growth \& Design, 1, 87-99.

http://dx.doi.org/10.1021/cg005511b

Lan C. W., Tu C. Y., 2002, "Three-dimensional Analysis of Flow and Segregation Control by Slow Rotation for Bridgeman Crystal Growth in Microgravity," Journal of Crystal Growth, 237-239, 1881-1885.

http://dx.doi.org/10.1016/S0022-0248(01)02210-2

Li Z., Yang M. and Zhang Y., 2014, "A Coupled Lattice Boltzmann and Finite Volume Method for Natural Convection Simulation," International Journal of Heat and Mass Transfer, 70, 864-874.

http://dx.doi.org/10.1016/j.ijheatmasstransfer.2013.11.077

Lima D., Wit De A., 2004, "Convective Instability in Protein Crystal Growth" Physical Review A, 70(2), 021603.

http://dx.doi.org/10.1103/PhysRevA.70.021603

Lin H., Rosenberger, F., Alexander, J.I.D., Nadarajah, A., 1995, "Convective-Diffusive Transport in Protein Crystal Growth," Journal of Crystal Growth, 151, 153-162.

http://dx.doi.org/10.1016/0022-0248(95)00016-X

Nanev C. N., Penkova A., and Chayen N., 2004, "Effects of BuoyancyDriven Convection on Nucleation and Growth of Protein Crystals," Ann NY Acad Sci, 1027, 1-9.

\section{http://dx.doi.org/10.1196/annals.1324.001}

Qi J., Wakayama N. I., 2000, "Solute Convection during the Whole Process of Protein Crystal Growth," Journal of Crystal Growth, 219, 465-476.

http://dx.doi.org/10.1016/S0022-0248(00)00658-8

Ramachandran N., Baugher C. R., and Naumann R. J., 1995, "Modeling Flows and Transport in Protein Crystal Growth," Microgravity Science Technology, 8(3), 170-179.

Riahi D., Obare C. W., 2009, "Analysis of Rotating Flow around a Growing Protein Crystal," Applications and Applied Mathematics: An International Journal, 4(1), 13-25.

Szurgot M., Sangwal K., 1987, "Influence of Free Convection on the Formation of Growth Defects in Potassium Bichromate Crystals Grown from Aqueous Solutions," Crystal Research and Technology, 22(12), 1477-1484.

http://dx.doi.org/10.1002/crat.2170221208

Simic-Stefani S., Kawaji M., Hu H. H., 2006, “G-jitter-induced Motion of a Protein Crystal under Microgravity," Journal of crystal growth, 294, 373-384.

http://dx.doi.org/10.1016/j.jcrysgro.2006.06.032

Takahashi S., Ohta K., and Furubayashi N., 2013, "JAXA Protein Crystallization in Space: Ongoing Improvements for Growing HighQuality Crystals," Journal of Synchrotron Radiation, 20, 968-973.

http://dx.doi.org/10.1107/S0909049513021596

Tanaka H. et al, 2013, "Numerical Model of Protein Crystal Growth in a Diffusive Field such as the Microgravity Environment," Journal of Synchrotron Radiation, 20, 1003-1009.

http://dx.doi.org/10.1107/S0909049513022784

Vekilov P. G. et al, 1999, "Nonlinear Dynamics of Layer Growth and Consequences for Protein Crystal Perfection," Journal of Crystal Growth, 196, 261-275.

http://dx.doi.org/10.1016/S0022-0248(98)00840-9

Vekilov P. G., Thomas B. R., and Rosenberger F., 1998, "Effects of Convective Solute and Impurity Transport on Protein Crystal Growth," Journal of Physical Chemistry B, 102, 5208-5216.

http://dx.doi.org/10.1021/jp973123n

Vekilov P. G., 1999, "Protein Crystal Growth-Microgravity Aspect," Advances in Space Research," 24(10), 1231-1240.

http://dx.doi.org/10.1016/S0273-1177(99)00725-5

Verma S., Srivastava A., and Prabhakar V., 2005, "Simulation and Experimental Verification of Solutal Convection in the Initial Stages of Crystal Growth from an Aquous Solution," Indian Journal of Pure and Applied Physics, 43, 24-33.

Wang L. B., Wakayama N. I., Tao W. Q., 2008, "The Role of Solutal Convection in Protein Crystal Growth-A New Dimensionless Number to Evaluate the Effects of Convection on Protein Crystal Growth," Journal of Crystal Growth, 310, 5370-5374.

http://dx.doi.org/10.1016/j.jcrysgro.2008.09.125

Xue D. T., Lei, J., Cheng, Y., 2004, "Microgravity Measurement of Spaceships Shenzhou" Physics, 33(5), 351-358 (in Chinese).

Yuferev, V.S., Kolesnikova, E.N., 1995, "Combined Action of Microgravitation and the Coriolis Force on the Motion of a Liquid in the Middle Part of a Three-dimensional Thin Rectangular Region," Technical Physics Letters, 21, 178-179.

Yuferev, V. S., and Kolesnikova, E. N., 1995, "The effect of the Coriolis Force on the Convection of a Liquid under Weightless Conditions in the Presence of Angular and Linear Oscillations of an Orbital Station about its Center of Mass," Technical Physics Letters, 21, 500-502. 